\title{
Inlet Conditions for LES using Mapping and Feedback Control
}

\author{
M.H.Baba-Ahmadi ${ }^{a}$ G.Tabor ${ }^{\text {a }} 1$ \\ a SECaM, University of Exeter, Harrison Building, North Park Road, Exeter, UK, \\ $E X 44 Q F$
}

\begin{abstract}
Generating effective and efficient inlet boundary conditions for Large Eddy Simulation (LES) is a challenging problem. The most accurate way of achieving this is to run a precursor calculation to generate a library of turbulence, either prior to the simulation or concurrently with it, and to transfer the data from the library simulation to the main domain inlet. In this paper we investigate a variant of this, in which the precursor calculation is subsumed into the main domain, its function being adopted by a mapping of data from a specified plane downstream of the inlet back to the inlet. Within this inlet section of the main domain, the flow can be affected by a number of computational manipulations, including the introduction of artificial body forces, modification of the mapped data, and direct correction of the velocity data. These modifications can be linked to feedback control algorithms to drive the solution towards specified characteristics, including mean and turbulent flow profiles, and bulk properties of the flow such as swirl. Various variants of the basic technique incorporating different levels of complexity in the control are implemented and tested on simulation of flow in a rectangular channel and in a circular pipe.
\end{abstract}

Key words: Large Eddy Simulation; Inlet conditions; CFD

\section{Introduction}

Turbulence is a complex state of fluid motion, usually described in terms of pseudo-random coherent motions on a range of spatial and temporal scales superimposed on some 'mean' flow. The range of motions is fairly continuous between large scales determined by the geometry of the problem and small scales determined by the viscosity of the fluid, and this range is sufficiently

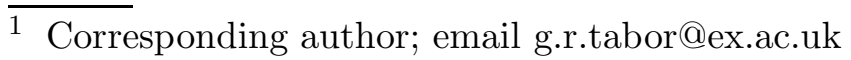


wide that for most cases of interest, direct numerical simulation of the full Navier-Stokes equations is impossible. Hence to solve turbulent flow problems we rely on turbulence modelling techniques, in which the number of degrees of freedom of the problem is substantially reduced by applying some averaging process : the resulting averaged Navier Stokes equations are then solved together with modelled equations for quantities representative of the fluctuation around this average. Hence in Reynolds Averaged Navier Stokes (RANS) methods, an ensemble average is applied to the flow to divide it into a mean flow and fluctuations around this mean, which we choose to label as 'turbulence'. The RANS equations are then solved together with equations for quantities such as the turbulent kinetic energy $k$ which are expected to be universal. Implicit within this method is the assumption of a scale separation between 'turbulent' and 'non-turbulent' scales in the flow. In practice we often take the ensemble average to be equivalent to a time-average, and assume that the timescale for the fluctuations around the mean that we are labelling as 'turbulence' is considerably shorter than any timescale for variation of the mean flow, which may in fact be time-independent.

An alternative approach to turbulence modelling, which is gaining in popularity as increasing computational power makes it more accessible, is that of Large Eddy Simulation (LES). Here the averaging applied to the flow is a spatial averaging in the form of a convolution with a spatial filter $G$, separating the flow into grid scale (GS) and sub-grid scale (SGS) components $\mathbf{u}=\overline{\mathbf{u}}+\overline{\mathbf{u}^{\prime}}$, where $\overline{\mathbf{u}}=G * \mathbf{u}=\int_{D} G(\zeta, \Delta) \mathbf{u}(\zeta, t) d^{3} \zeta, \Delta$ is a characteristic scale of $G$, referred to as the filter width, and $D$ is the computational domain. Conventionally we assume that the filter width is the same as the cell size $\Delta x$, hence the labels Grid Scale and Sub-Grid Scale. In this case the averaged, or filtered Navier-Stokes equations take the form

$$
\begin{aligned}
& \nabla \cdot \overline{\mathbf{u}}=0, \\
& \partial_{t} \overline{\mathbf{u}}+\nabla \cdot(\overline{\mathbf{u}} \otimes \overline{\mathbf{u}})=\nabla \cdot(\overline{\mathbf{S}}-\mathbf{B}),
\end{aligned}
$$

given that the differential operators commute with the filtering, i.e. $[G *, \nabla] \mathbf{u}=$ $\mathbf{0}[6]: \mathbf{u}$ is the velocity field, $\nu$ the molecular viscosity, $\mathbf{S}=-p \mathbf{I}+2 \nu \mathbf{D}$ with $p$ the specific pressure, and $\mathbf{D}=\frac{1}{2}\left(\nabla \mathbf{u}+\nabla \mathbf{u}^{T}\right)$. The convolution process generates an additional term, the SGS stress tensor :

$$
\mathbf{B}=\overline{\mathbf{u} \otimes \mathbf{u}}-\overline{\mathbf{u}}_{\otimes} \overline{\mathbf{u}}=\mathbf{L}+\mathbf{C}+\mathbf{R}
$$

where $\mathbf{L}$ is the Leonard stress, $\mathbf{C}$ the cross stress and $\mathbf{R}$ the Reynolds stress tensor (e.g. [8]). Different modelling of these terms generates the different turbulence models.

There are several advantages of adopting this formulation. In particular, no assumption is being made concerning scale separation. In fact, it is commonly 
assumed that the energy in the motions in the flow is continuously distributed in the conventional $-5 / 3$ power law spectrum of turbulence, and this energy spectrum is being truncated somewhere in the turbulent cascade, with the larger turbulent scales being explicitly simulated whilst the smaller, more universal scales are being replaced by the SGS turbulence model. Since we are often interested in the effects of the larger scales of the turbulence (but less often in the very smallest scales), then this leads to a more accurate representation of the transitory component of the flow, irrespective of whether we choose to label it 'turbulence' or not. This can be valuable in cases such as turbulent combustion [29] where additional physics is linked to the actual (fluctuation) flow, not simply the 'mean' flow. However it does present problems when we need to apply boundary conditions. In RANS, because of the scale separation, all quantities specified on boundaries are constants, or at most slowly varying in time in comparison with the simulation timestep. In LES however, the GS variables always include some time-varying component, stochastically varying on all scales down to the spatial scale (the grid scale $\Delta x$ ) and the temporal scale (the timestep $\Delta t$ ) of the simulation. Put simply, at an inlet turbulent fluctuations are present on the grid scale, and some method must be found for generating stochastic fluctuations in the grid scale quantities that 'look' like turbulence. Although in some problems (such as bluff-body flows), turbulence at the inlet is not a significant contribution to the turbulence within the domain, in a lot of cases the inlet conditions will have a significant impact on the flow dynamics, and thus the correct implementation of inlet conditions is of significant importance.

There are basically two approaches available for generating inlet conditions for LES; library lookup, in which a separate calculation of turbulent flow is performed for a simpler case and mined to extract information for the inlet, and synthesis methods, in which random fluctuations are generated at the inlet with specific numerical character, e.g. specified correlation lengths or Gaussian distributions $[17,24,19,16]$. Synthesis techniques have some advantages, but because of the problems with generating correlations between locations and components, can only ever provide an approximation of turbulence at the inlet. This approximation serves to trigger the development of true turbulence, and a section of domain must be provided for this to develop its full character. Library lookup techniques use genuine turbulence, and thus in general the flow entering the domain is computationally correct; no further development section is necessary. However there is the issue of how to generate the library to the correct specifications, and how to store and manipulate the potentially large quantity of data necessary for the simulation. Typically the library is created by simulating a simple precursor case, eg. a short cyclic duct [8] which can be manipulated in various ways in order to affect its velocity profile $[25$, 26,27 ] or to introduce mean flow conditions such as swirl [20]. The library generation can be run as a precursor calculation, in which case there is the problem of storage of the data to be used and its recalculation (or in some cases 
manipulation [22]) if the turbulence conditions alter. Alternatively the library generation can be run concurrently with the main simulation $[18,4]$, which avoids the problem of storing a large but finite database of turbulence, but does involve simultaneous simulation on separate meshes. Comparisons between the different techniques - particularly between synthesis and library techniques - are rare, but have been published for the case of flow in a rectangular channel $[15,30]$.

In this paper we investigate a variant of the library lookup technique, in which flow properties are sampled on a plane downstream of the inlet and mapped back to the inlet. A similar method was adopted by Lund, Wu and Squires [18], in which two computational domains were used; a shorter precursor domain in which data from the centre plane is mapped (with some modification) back to the inlet; the same data is also mapped from this plane to the inlet of the main (much longer) computational domain. However there is no intrinsic reason why two domains need to be used for this. Hence in this paper, the mapping takes place on the main domain $[31,30]$ and no separate precursor calculation is necessary. The simple procedure of mapping the data back to the inlet will create a section of the main mesh where fully developed turbulent flow develops. However in many cases we would not want fully developed flow but turbulent flow that matches some predefined parameters, such as a known turbulence profile or bulk swirl. This can be achieved by modifying the flow, either by introducing artificial body force terms into this section, or by explicit correction of the velocities within this section, or by suitable modification of the mapped data. By linking these techniques to a feedback control algorithm based on the discrepancy between computed values and desired values at a given location in the flow, it is possible to drive the flow towards the required flow conditions. Several of these techniques have been introduced before in other contexts, for example Pierce and Moin's [20] methodology for generating swirl in a cyclic cylindrical domain using a constant body force. Here we combine these different elements to create a methodology for generating inlet conditions which is elegant, efficient, easily modifiable, and which generates genuine turbulent flow without the need for a development section of the mesh, since even the flow in the mapped section is genuinely turbulent.

The format of the paper is as follows. In section 2 we introduce the details of the method and its variants, together with numerical details of the code used for the demonstration cases. In section 3, results for the most basic implementation are compared with experimental and DNS data for the case of channel flow between two flat plates. Then in section 4, the case of flow in a circular pipe is considered. Successively more complex variants of the basic method with more refined correction and control methodology are used to compute flow for this case and compared with internally generated results for a short cyclic pipe, and with literature data. The accuracy of the results and the impact of the mapping and flow correction techniques on the flow within 
and downstream of the mapping section are investigated to demonstrate the accuracy of the basic technique, and to compare variants.

\section{Computational Methodology}

\subsection{Basic mapping method - Method A}

As briefly explained above, existing library lookup methods consist of running a separate, precursor case to generate turbulence data. This typically involves a calculation on a cyclic domain, or one where data is sampled at an interior plane and mapped back to the inlet. Then, the velocity field in one plane normal to the streamwise direction is stored at each time step. The sequence of planes is then read in as inflow data for a separate calculation of the flow of interest. However there is no reason why the mapping has to be performed on a separate flow case rather than on the main domain itself. This eliminates the need for running a separate case and in turn reduces the cost of calculation both in terms of computational time and memory requirement. In this case, by mapping flow data from a cutting plane somewhere in the body of the computational domain, an inlet section is created in which the flow is forced to become fully-developed. At the same time, the flow conditions within this section are automatically fed into the main domain.

There remain two questions to answer here; what properties of the flow need to be mapped to the inlet, and how to drive the flow within the mapping section to create a non-zero and specified flow in this region? Clearly, since there are dissipative processes occurring within the mapping section (viscous dissipation and wall friction), if the flow parameters are mapped back unchanged the total flow through the section, and thus through the domain as a whole, will eventually drop to zero. To avoid this, the velocities being mapped must be corrected to ensure a specified inlet flux of fluid $Q_{i n, 0}$ The Method of Characteristics [5] can be used to determine the number of variables to be specified at particular boundaries in a CFD simulation; specify too many or too few and the resulting system of equations will be over- or under-determined, respectively. For an inlet condition, this typically translates into a specification of the inlet velocity normal to the boundary; although this can also be specified as an inlet flux or as an inlet pressure (which will then be converted to an equivalent velocity and flux). Thus the simplest approach will be to map the GS velocity from the sampling plane back to the inlet, scaling this by a multiplicative factor $Q_{i n, 0} / Q_{i n}$ to ensure the desired integrated inlet flux is always achieved. If the pressure field $p$ is mapped then this can be converted to a velocity field which can then be similarly scaled. Continuity applied to the inlet section will then guarantee the maintenance of the flow through the 
inlet section - if an amount of fluid $Q_{i n, 0}$ is always entering the domain, the same amount must always be flowing through it. The SGS turbulence properties will also be mapped to the inlet; for the 1-equation eddy viscosity model being used here, this involves mapping the SGS $k$ back to the inlet. $k$ should be a balance between generation (from the walls and across the filter cut-off from the GS turbulence) and viscous dissipation, and so should stabilise at a physical level.

\subsection{Mapping with axial body force - Method B}

Method A represents the simplest form of mapping that can be introduced, and also the weakest feedback system providing control over the minimum number of parameters of the flow (basically just the inlet flux). In order to improve its performance, and in particular to allow other parameters of the flow to be controlled, we need to introduce further forcing mechanisms into the system. This can be done by applying a virtual body force to the flow in the mapping section, whose characteristics (magnitude and direction) can be controlled to achieve the desired result. In method B, inflow conditions are constructed using axial body forces inside the mapping section, whilst at the same time transferring data from the mapping surface to the inlet boundary. The momentum equation in (1) now includes a body force term :

$$
\partial_{t} \overline{\mathbf{u}}+\nabla \cdot(\overline{\mathbf{u}} \otimes \overline{\mathbf{u}})=\nabla \cdot(\overline{\mathbf{S}}-\mathbf{B})+\overline{\mathbf{F}},
$$

where $\overline{\mathbf{F}}$ is an artificial axial body force of the form

$$
\overline{\mathbf{F}}=\underline{k} F_{z}
$$

with $F_{z}=\frac{\partial p}{\partial z}$. This axial body force added to the momentum equations is applied just to the mapping section and represents the mean pressure gradient that drives the flow inside the mapping section. Because of the stronger forcing in the system, deviation from the desired flow is corrected faster than in method $\mathrm{A}$, and thus the dependency of this method on initial conditions should be less than for the previous case. Since the force is restricted to be entirely axial, this method is only applicable for fully developed parallel flow.

\subsection{Explicit Velocity Correction - Method C}

The introduction of axial body forces alone is not necessarily enough to generate the desired mean velocity profiles exactly and there are found to be some discrepancies from the target profiles. Therefore, as a second level of control, a velocity correction relation is introduced for each velocity component. 


$$
\overline{\mathbf{u}}^{*}=\overline{\mathbf{u}}+\left(\mathbf{u}_{d e s}-\widehat{\overline{\mathbf{u}}}\right)
$$

and the GS velocities $\overline{\mathbf{u}}$ replaced with these corrected velocities $\overline{\mathbf{u}}^{*}$ for all cells in the inlet mapping section. Here, $\mathbf{u}_{\text {des }}$ is the target mean flow profile and $\overline{\mathbf{u}}$ the instantaneous GS velocity, whilst $\widehat{\overline{\mathbf{u}}}$ is the time average of the GS velocity, evaluated by taking a running average on the GS velocity. The correction term in this relation approaches zero as the the sampled velocity profile $\widehat{\overline{\mathbf{u}}}$ approaches the desired velocity profile $\mathbf{u}_{\text {des }}$. Hence this correction assists the convergence to the desired mean velocity profile and reduces the number of timesteps needed to achieve the desired result.

\subsection{Virtual axial and azimuthal body forces - Method D}

To apply the Mapping method to spatially developing turbulent flows such as swirling flows, further modifications are needed. For these cases, all velocity components are important and should be considered and manipulated in the mapping section. Hence the artificial body forces added to momentum equations should be in three dimensions. Method $\mathrm{D}$ extends the virtual body force used in method B, using a control algorithm to generate the correct 3-d mean flow, but also includes a modified version of the velocity correction method of method $\mathrm{C}$ in order to generate specified turbulence profiles.

Pierce and Moin [20] developed a method for generating swirling flow in a periodic domain in which virtual axial and azimuthal body forces are added to the momentum equations. The axial force represents the mean pressure gradient that drives the flow in the mapping section, whilst the azimuthal body force overcomes the drag from walls and drives the azimuthal flow. Pierce and Moin used this as the basis for a library lookup method to generate swirling inlet flow [20] which was then used as an inlet flow for a confined coaxial jet. Method D uses a similar approach, with two differences. As before, the inlet generation is embedded in the main domain rather than applied to a separate computation. Also, in Pierce and Moin's method, the body forces are selected as constant, linear and/or quadratic functions of radial locations and are independent of velocity conditions, whereas in the present work, the body forces are functions of the velocity conditions in the mapping section as defined below.

Again, the modified governing equations (3) are used, but now with the additional body force $\overline{\mathbf{F}}$ defined as

$$
\overline{\mathbf{F}}=\frac{U_{b}}{L}\left[\alpha\left(\mathbf{u}_{\text {des }}-\widehat{\mathbf{u}}\right)+\left(\mathbf{u}_{\text {des }}-\overline{\mathbf{u}}\right)\right],
$$

inside the mapping section and $F=0$ outside the mapping section (the main 
flow). Here $U_{b}$ and $L$ are the bulk velocity and the length of the forcing region respectively. In equation (6), the first term in the bracket i.e. $\alpha\left(\mathbf{u}_{\text {des }}-\widehat{\overline{\mathbf{u}}}\right)$ provides feedback control, with $\alpha$ setting the magnitude of the feedback. However, early in the simulation $\mathbf{u}_{\text {des }}-\widehat{\mathbf{u}}$ is large, with potentially unfortunate consequences. Thus at the start of the simulation, the value $\alpha=0$ is chosen. As the simulation progresses $\mathbf{u}_{\text {des }}-\widehat{\overline{\mathbf{u}}}$ decreases towards zero, so the value of $\alpha$ can be increased. To achieve this, $\alpha$ is set equal to the timestep number, thus ramping its value as the simulation progresses. The deviation of each individual component of the time-averaged GS velocity $\widehat{\hat{\mathbf{u}}}$ is monitored, and whilst $\left|\mathbf{u}_{\text {des }}-\widehat{\hat{\mathbf{u}}}\right| / U_{b}>0.0001$ the value of $\alpha$ is increased linearly with timestep number. In any case the maximum value of $\alpha$ is limited to $\alpha=2000$. The reason for these two limiting procedures is that there will always be a discrepancy between the target and computed mean flow velocities at some points in the mesh. Thus $\mathbf{u}_{\text {des }}-\widehat{\hat{\mathbf{u}}} \neq 0$. If $\alpha$ was allowed to increase without limit, the force contribution from this term would eventually come to dominate the equation for no physical reason, and the simulation would fail.

This body force/feedback control system generating the mean flow is supplemented by a modified velocity correction procedure designed to drive the flow towards the correct turbulence flow profile. The new velocity correction term takes the form

$$
\overline{\mathbf{u}}^{*}=\mathbf{u}_{d e s}+(\overline{\mathbf{u}}-\widehat{\hat{\mathbf{u}}}) \times\left(\frac{\left(\tau_{d e s}\right)_{i i}}{\tau_{i i}}\right)^{1 / 2},
$$

where $\tau_{\text {des }}$ is the desired Reynolds stress and $\tau$ is the calculated Reynolds stress in the mapping section. Here Einstein summation convention is not used; $\tau_{i i} \equiv \tau_{11}$ or $\tau_{22}$ or $\tau_{33}$ according to which velocity component is being corrected. To explain the relation we observe that:

$$
\left(\frac{\left(\tau_{d e s}\right)_{i i}}{\tau_{i i}}\right)^{1 / 2}=\frac{\sqrt{\overline{{u_{i d e s}^{\prime 2}}_{i}}}}{\sqrt{\overline{{u_{i}^{\prime 2}}_{i}}}}
$$

Because of the fluctuations, the term $(\overline{\mathbf{u}}-\widehat{\mathbf{u}})$ is never exactly zero, and so the term $\left(\frac{\left(\tau_{d e s}\right)_{i i}}{\tau_{i i}}\right)^{1 / 2}$ drives the turbulent fluctuations to provide the target root mean square velocity components and in turn the target axial components of the Reynolds stress. This can be seen more clearly by rearranging Eqn.(7). Subtracting $\mathbf{u}_{\text {des }}$ from both sides gives

$$
\mathbf{u}^{*}-\mathbf{u}_{d e s}=(\overline{\mathbf{u}}-\widehat{\overline{\mathbf{u}}}) \times\left(\frac{\left(\tau_{d e s}\right)_{i i}}{\tau_{i i}}\right)^{1 / 2},
$$

Now squaring both sides and averaging, 


$$
\left\langle\left(\overline{\mathbf{u}}^{*}-\mathbf{u}_{d e s}\right)^{2}\right\rangle=\left\langle(\overline{\mathbf{u}}-\widehat{\overline{\mathbf{u}}})^{2}\right\rangle \times\left(\frac{\left(\tau_{d e s}\right)_{i i}}{\tau_{i i}}\right)
$$

The terms $\left(\overline{\mathbf{u}}^{*}-\widehat{\mathbf{u}}_{d e s}\right)^{2}$ and $(\overline{\mathbf{u}}-\widehat{\widehat{\mathbf{u}}})^{2}$ represent velocity fluctuations around the mean which are the basis of the Reynolds stresses, and thus we see that they are being scaled by the ratio of the desired turbulence level to the sampled turbulence level. This relation was first implemented in [22] to generate a database for lookup based on an a priori RANS calculation; here it is applied directly to control the flow.

\subsection{Computational Details}

The filtered Navier-Stokes equations, (1) together with (2), are solved using the CFD code library OpenFOAM. This is a $\mathrm{C}++$ code library of classes for writing CFD codes, which includes a well-tested and validated LES capability $[8,7,9,10]$. Equations (1) are discretised using the finite volume method, where the domain $D$ is divided into cells $\delta V_{i}$ so that $\bigcup_{i}\left(\delta V_{i}\right)=D \cup \partial D$ and $\bigcap_{i}\left(\delta V_{i}\right)=\emptyset$. Integration of the dependent variables over each cell $\delta V_{i}$, together with application of Gauss' theorem, generates a set of discretised equations with the divergence terms in Eqn. (1) represented as fluxes across the cell faces, evaluated using appropriate interpolation schemes; we use centred second order interpolation and NVD-derived interpolation (gamma scheme, see [14]). Time integration is carried out by the Crank-Nicholson scheme, which is 2nd order in time. Following the procedure of Rhie and Chow [21], discretisation of the $\nabla p$ term is left; a Poisson equation is constructed which implements the incompressibility condition $\nabla \cdot \overline{\mathbf{v}}=0$, and the equation set solved sequentially using the resulting PISO algorithm [12]. Solution is performed implicitly by matrix inversion using Incomplete Cholesky Conjugate Gradient methods. SGS modelling is provided by the dynamic 1-equation model, in which a transport equation is provided for the sub-grid turbulent kinetic energy $k$, and the resulting model coefficients can be determined by introducing a second, gridscale level of filtering [11]. The one-equation approach may allow for coarser grids than can be used for a comparable problem with a zero-equation model because some sub-grid information is available for the formulation of sub-grid scale models. All of this has been implemented in OpenFOAM previously and extensively validated [8].

\section{Channel flow case}

As a preliminary test case, method A is applied to simulate fully-developed turbulent flow in a channel bounded by infinite walls at $y= \pm d$. This is a 
typical test case for which a wide range of data is available; We compare with DNS data published by the Kasagi-Suzuki/Shikazono Lab [13] which provides profiles of velocity and higher moments for various Reynolds numbers, and with LES data from shorter, cyclic channels [8]. The geometry consists of a domain of $20 \mathrm{~m}$ in length between two flat plates $2 \mathrm{~m}$ apart, as shown in figure 1; the channel is therefore 10 channel-widths or 7900 wall units in length. The mean flow velocity and fluid viscosity are arranged to create a flow at Reynolds number $\mathcal{R} e=13750$ based on the channel half-width, equivalent to a friction Reynolds number $\mathcal{R} e_{\tau}=400$ based on the friction velocity. The flow is in the $x$-direction, and the domain boundaries are symmetry planes at $z=0$ and $z=2 \mathrm{~m}$, giving (in effect) an infinite domain in this direction. The mesh is generated from two blocks in the $y$ direction, allowing mesh grading towards the walls coupled with van Driest damping to deal with the near-wall flow. The basic mesh resolution is $60 \times 50 \times 30(x \times y \times z)=90,000$ cells, with the first near-wall layer of cells being of width $\Delta y^{+}=2$, a resolution which has been found to give good results [7]. Outlet conditions were simply implemented as zero gradient for velocity and $k$, and fixed value for pressure.

Figure 2 shows time-averaged mean velocity and fluctuating velocity components plotted as profiles against distance from the wall, for various locations away from the inlet. The mean velocity profile plus the primary components of the grid scale turbulent stress are plotted, where

$$
\widehat{T_{i j}}=\widehat{u_{i}^{\prime} u_{j}^{\prime}} \quad \text { and } \quad u_{i}^{\prime}=u_{i}-\widehat{u_{i}}
$$

so these represent the streamwise, cross, and spanwise turbulent stresses. Also shown are results from the literature DNS reference data [13], and from our own cyclic channel LES calculation. The matrix of results shows graphs at various points along the duct, from $x / d=1$, close to the inlet itself, to $x / d=$ 20 which is at the outlet. The mean velocity profile generated is close to the DNS data, and little evolution is seen downstream of the inlet. Of greater importance is the reproduction and evolution of the streamwise, spanwise and cross components of the stress. The simple mapping method is generating the $T_{x x}$ and $T_{x y}$ components well. The shape of the $T_{y y}$ profiles is well reproduced, but the magnitude is over-predicted, at least initially.

To evaluate effects of mesh size on the precision of results for a long channel, the same method was used to generate inlet conditions for LES of turbulent flow for the same long channel with different resolutions in the $x, y, z$ directions. Figure 3 shows the effect of mesh sizes on accuracy of results in comparison with results obtained from the short cyclic channel. As can be seen the stress profiles improve as the mesh is refined; on the highest resolution mesh there is a very good agreement between results obtained by the Mapping method compared to the cyclic channel. 


\section{Pipe flow}

In this section, methods A-D are applied to LES of parallel pipe flow. Accurate DNS data for turbulent pipe flow is much less common than for the parallel plate case [32], although some DNS and experimental data is available in the literature $[32,23]$. For comparisons between methods A-D, results generated by a cyclic short pipe for the same flow conditions and the same grid structure are calculated and used as reference data. In addition the results from this preliminary computation are used to provide target profiles $\mathbf{u}_{\text {des }}$ and $\tau_{\text {des }}$ for the control algorithm to aim at. Flow data such as mean velocity profile, Reynolds stress profiles, enstrophy, vorticity and time series spectra are presented and discussed. The validation test case is a turbulent parallel pipe flow with Reynolds number $R e=30,000$ where Reynolds number is calculated based on diameter of the pipe $D$. A fairly coarse mesh was used with 2176 cells covering the cross section and 10 cells per meter of length in the $z$-direction $(435,200$ cells in total). The short cyclic pipe reference data had the same mesh structure in cross section but used 196, 800 cells in total. Very recently a study of turbulent pipe flow at $\mathcal{R} e=44,000$ has been published by $\mathrm{Wu}$ and Moin [33], and a further computation has been performed comparing our method D and the short cyclic reference flow data with this data. The same mesh was used for the long pipe as for the previous case, with the DNS data used as the target and for comparison at various points along the pipe.

Since in the Mapping method, all flow conditions generated in the mapping section are automatically fed to the main flow, it is necessary to compare those with flow conditions in the main domain and reference data to check them. Before discussing the results, it is necessary to clarify what will be regarded as the inlet and what the main section of the computational domain. In method A, no additional terms are introduced into the equations or to modify the flow, so there is no reason why the mapping cannot take place within the flow analysis section of the mesh. However, in methods B, C and D some additional terms are added to the governing equations and also, for methods $\mathrm{C}$ and $\mathrm{D}$, the velocity components are corrected to get the target velocity profiles. We are interested in the impact of these changes on the flow, and so for this study we wish to distinguish between the flow in the mapped section where the additional terms are included, and the flow downstream where the flow is allowed to evolve naturally. The geometry used here for the comparisons consists of a pipe with the same mesh density and cross section but different length for various methods. For the reference cyclic short pipe, the geometry consists of a pipe of diameter $D=2 \mathrm{~m}$ and length $2 D$. For method A, the length of the pipe is $10 D$, whilst for the other methods (B, C and D) this length is $12 D$, thus providing a $10 D$ main domain and a $2 D$ mapping section. For all cases then, there is a $10 D$ section in which the flow is allowed to evolve naturally and which is completely comparable. To evaluate effects of 
the additional artificial forces and velocity corrections on the flow, the flow conditions for the mapping section are presented as well and compared with those in the main domain and with results from the cyclic short pipe. The structure of the mesh is shown in figure (4), with a central rectangular block surrounded by four blocks adapting to the cylindrical outside of the domain; this eliminates any singular mesh behaviour on the central axis of the domain. The same outlet conditions were used as for the channel flow calculation.

\subsection{Comparison of Flow Profiles}

Figure 5 shows the mean velocity profiles for the methods A-D at 7 different locations along the pipe. The graphs show excellent agreement with reference data for all methods. Figures 6 - 9 show Reynolds stress components $\tau_{z z}, \tau_{r r}, \tau_{\theta \theta}$ and $\tau_{z r}$ respectively. As can be seen the graphs show a very good agreement for $\tau_{z z}, \tau_{r r}$ and $\tau_{\theta \theta}$ for methods $\mathrm{A}, \mathrm{B}$ and $\mathrm{D}$. The graphs related to method $\mathrm{C}$ also shows there is no significant negative effect on the flow due to the use of the velocity correction relations. Figure 9 presents the results for $\tau_{z r}$. The graphs show good agreement for methods A and B, although there are weak results for method D around the inlet. This can be explained by referring to Eqn.(7). Since the chosen parameters for rescaling various velocity components are different, so the cross correlations between the velocity components are destroyed whilst the autocorrelations are saved during the inflow generation process. An interesting point however, as can be seen in the graphs, is that the cross correlations approach to the real values rapidly downstream of the inlet. The results presented here indicate that if turbulent fluctuations are generated accurately at the inflow, weak cross correlations rapidly improve to correct values downstream of the inlet without producing any significant negative effects on the main flow.

Results at the higher Reynolds number are shown in figure 10. at various locations along the pipe. The mean flow profile is reasonably well reproduced for all sections along the pipe. For the turbulent parameters, the profiles sampled close to the mapping plane are very well reproduced indeed, although the solution shows a tendency to evolve away from the DNS solution further downstream from the mapping section near the wall $\left(\tau_{z z}\right.$ and $\left.\tau_{r z}\right)$ and across the flow $\left(\tau_{r r}\right.$ particularly). The change is not large and it stabilises further downstream; it is likely that there are problems with the near-wall mesh resolution which are causing this effect. We suggest that given suitable near-wall resolution and/or better wall modelling we would be able to obtain much better agreement with the DNS reference data. However, the intention in this paper is to investigate the behaviour of the inlet conditions rather than achieve the highest possible accuracy of the LES simulation itself. With this in mind, the other point to note is that the LES profiles stabilise after $z / D=2.5$ 
and remain essentially unchanged with distance down the pipe; again the inlet is generating turbulent flow structures which behave correctly right from the inlet; there is no need for any adaption of the inlet flow as is the case for synthesis methods.

\subsection{Time-series spectra}

Time series energy spectra for the different methods are presented in figures 1112, showing large eddy behaviour at low frequency, a power law section and a steep drop at the filter cut-off. This drop off is a feature of the FV LES numerics rather than any issue relating to the inlet conditions. The filter width as well as the details of the filter shape are free parameters in LES and these can be used both to control the effective resolution of the simulation and to establish the relative importance of different portions of the resolved spectrum. However due to the lack of a straightforward and robust filtering procedure for inhomogeneous flows, most large eddy simulations performed to date have not made use of explicit filtering. The nearly universal approach for LES in complex geometries is to argue that the finite support of the computational mesh together with the low pass characteristics of the discrete differencing operators, effectively act as a filter. This procedure is typically referred to as implicit filtering since an explicit filtering operation never appears in the solution procedure. In finite volume methods where volume averaging is used to determine properties at cell centres, the effect of the implicit filter can be represented by the top-hat filter [1].

De Stefano et al. [28] showed that in explicit filtering LES, the subgrid scale stress tensor strongly depends on the assumed filter shape, which causes the SGS model to be filter dependent. Depending on the choice of the filter, the corresponding model should satisfy very different requirements in terms of large scale dynamics and kinetic energy budget. In the same reference they showed that the application of a smooth filter such as the top hat filter strongly affects the shape of the energy spectrum. In other words, for this kind of filter, even when LES is conducted with the ideal SGS model, the resolved field loses some important features of the real field. In particular, the slope corresponding to the inertial range is clearly misrepresented. In their paper, they compared the energy spectra corresponding to LES using sharp cut-off filtering and top hat filtering. They showed for sharp cut-off filtering, there is a sharp cut-off in the energy spectrum while for top hat filtering there is a smooth decrease in energy spectrum for high frequencies.

Bearing this in mind, the smooth decrease in the energy spectrum at high frequencies which can be seen in figures 11-12 for methods $\mathrm{A}, \mathrm{B}$ and $\mathrm{C}$ is reasonable for finite volume method LES. However figure 12 shows different 
results for Method D. Here, for all sample locations downstream of the inlet except right close to the outlet, we see a sharp truncation of the energy spectrum. Since all flow conditions and mesh structure are the same for all test cases, this suggests that the difference may be related to the rescaling process used in this method. Since this rescaling process is a common approach used elsewhere, these results should be examined in more detail in future work.

\subsection{Enstrophy and Vorticity}

In this section the results for enstrophy and vorticity are presented for methods A-D. These contour plots present a qualitative comparison to examine the effects of the different elements of the different mapping methods. Since there are no additional terms or changes introduced to the governing equations for method A, in this comparison the results from method A are used as a baseline to evaluate results from the other methods. Figures 13.a. and 13.b. show contour plots of enstrophy and vorticity for the methods A-D respectively. The figures show qualitatively, in comparison with method A, that there is no significant negative effect due to the different control algorithms used in methods B-D. In particular, the plots do not show any significant difference between results before and after the mapping surface, and there is a steady flow from mapping section to main section. This can also be seen in figure 14 which plots level of enstrophy as a function of distance down the pipe for the various methods. This plot was produced by sampling the enstrophy field at a number of equivalent points at each $x$-location and averaging them. Although there is some degree of variation in the level, there is no overall trend for any of the methods, thus demonstrating that true turbulence is being generated at the inlet; no further processing is occurring in the domain, and there is no significant change in the level at or across the mapping plane.

\section{Discussion}

There are clearly a number of questions to be answered when introducing a new LES inlet condition. Here, the most significant questions are; how well does the condition reproduce the turbulence at the inlet, how easy is it to implement and manipulate, and what impact do the correction and control elements have on the flow. The results presented here suggest that the simple baseline technique, Method A (introduced by de Villiers [31] and similar to recycling techniques used elsewhere in auxiliary calculations to generate turbulence libraries [18]) performs very creditably on both channel flows and pipe flows. On its own this is highly suitable for generating inlet turbulence where a particular volumetric flow rate, but no additional information can 
be specified; by performing the mapping directly on the main computational mesh the method is simplified in implementation, and there is no evidence that this causes any problems with the flow in the mapped section or immediately downstream of the mapping plane. Mean and grid scale turbulent flow properties are all generated accurately or evolve rapidly towards the anticipated values, and turbulent spectra and enstrophy/vorticity seem well generated throughout the domain.

Methods B-D are all variants of the basic method, where we have linked in correction and control elements in order to be able to specify desired flow and turbulence properties. Hence in Method B we introduce a constant axial body force which seems to aid convergence towards the desired volumetric flow. In Method $\mathrm{C}$ we introduce a velocity correction algorithm which updates the flow within the mapping section based on the error between the sampled profile and a desired mean flow profile. This enables us to control the flow to generate a required velocity profile. In Method $\mathrm{D}$ elements of both algorithms are combined, using a $3 \mathrm{~d}$ virtual body force with a control algorithm to generate a desired mean velocity profile, and a velocity correction combined with a control algorithm to generate a desired turbulence profile. In all three cases good results are produced on the parameters examined; mean and most turbulent quantities are well reproduced, and the turbulent energy spectrum is unaffected by the methodology used. Distinctions between the different methods are difficult to draw; the choice would be more predicated on the necessity to match available data. We do notice that the method $\mathrm{C}$ seems to enhance the turbulence in the domain more than the other methods; this is seen in figures 6-9. This may be due to the explicit velocity correction used in this method generating velocities which are no longer a direct solution of the governing equations; this may provide further random fluctuations around the actual solution which enhance the turbulence in the domain. As seen in figure 10 our underresolved LES is systematically underpredicting the turbulence, so this might fortuitously aid the solution. Care must be taken not to make the control algorithms too efficient; if the velocity control is too good then it can have the effect of completely destroying fluctuations in the velocity, thus unintentionally relaminarising the flow. Again, there is no evidence of significant change in flow properties between the mapped section and the main part of the flow, or across the mapping surface, despite the alteration of the mathematical system being simulated in the mapped section. Thus, although we have drawn a distinction between the two parts of the mesh in order to investigate this issue, this is probably not necessary; the additional terms in the equations will probably have little impact on the modelling except in cases where significant additional physics is involved, eg. in combustion. In most cases it would not be necessary to artificially lengthen the domain to incorporate the evolution of the inlet conditions, as is the case for synthesis methods [15].

There is the issue of where to obtain the target flow and turbulence pro- 
files. For simplicity we have used statistics garnered from our comparison LES simulations using cyclic domains, or from the target DNS, but in reality any source would do. The methodology could be applicable for Hybrid RANS/LES approaches where the Reynolds averaged flow statistics delivered by a RANS flow solver are used to guide the inflow conditions for a LES flow solver. In this case one would have to take care with the distinction between RANS and LES concepts of turbulence, and quite likely introduce assumptions about the anisotropy and/or length scale of the turbulence; however these are not insurmountable issues. In a similar way, experimental data could be used to define LES inflow conditions, and again this method could be used to incorporate the experimental conditions into the inlet flow. The bulk flow profiles may also involve significant large-scale motion such as swirl, something which we investigate elsewhere $[3,2]$; it is also plausible that this methodology could be adapted to introduce a low frequency, deterministic (i.e. non-turbulent) variation into the flow.

\section{Conclusions}

We present variants of a basic technique for generating inlet turbulence for LES calculations by mapping data from downstream back to the inlet; this mapping is performed on the main domain of the calculation, thus obviating the need for an auxiliary calculation to generate a turbulence library. The fluctuations coming into the domain behave like true turbulence, unlike synthesis methods where an adaption region is frequently necessary for actual turbulence to evolve. The basic method (Method A) allows the specification of the overall flow rate; in addition, various correction terms are introduced and linked to control algorithms allowing prescribed mean flow and turbulence profiles to be specified in a straightforward manner, something which other inlet techniques do not do. The resulting techniques are powerful, simple to manipulate, and perform well in all tested aspects.

\section{Acknowledgements}

We would like to thank Eugene de Villiers and Henry Weller for discussions about mapping inlets. This work was funded by the EPSRC under grant GR/R27495/01. 


\section{References}

[1] Adams NA, Kempe SHT, Domaradzki JA, Alternative approaches to large eddy simulation - implicit. ERCOFTAC 2005; pp 1 - 32

[2] Baba-Ahmadi MH 2008; Construction of inlet conditions for large eddy simulation. PhD thesis, SECaM, University of Exeter

[3] Baba-Ahmadi MH, Tabor G, Inlet conditions for LES of gas-turbine swirl injectors. AIAAJ 2008; 46(7):1782 - 1790

[4] Ferrante A, Elghobashi SE, A robust method for generating inflow conditions for direct simulations of spatially-developing boundary layers. J Comput Phys 2004; 198:372

[5] Fletcher CAJ 1991; Computational Techniques for Fluid Dynamics., Springer Series in Computational Physics, vol I\& II, 2nd edn. SpringerVerlag

[6] Fureby C, Tabor G, Mathematical and physical constraints on large eddy simulations. TheorCompFluid Dyn 1997; 9(2):75 - 83

[7] Fureby C, Gosman AD, Tabor G, Weller HG, Sandham N, Wolfshtein M 1997; Large eddy simulation of turbulent channel flows. In: Proceedings of Turbulent Shear Flows 11, vol 3, pp $28-13$

[8] Fureby C, Tabor G, Weller H, Gosman AD, A comparative study of sub grid scale models in homogeneous isotropic turbulence. PhysFluids 1997; 9(5):1416 - 1429

[9] Fureby C, Tabor G, Weller HG, Gosman AD, Differential subgrid stress models in large eddy simulations. PhysFluids 1997; 9(11):3578 - 3580

[10] Fureby C, Tabor G, Weller HG, Gosman AD, Large eddy simulation of the flow around a square prism. AIAAJ 2000; 38(3):442 - 452

[11] Ghosal S, Lund TS, Moin P, Akselvoll K, A dynamic localization model for large eddy simulation of turbulent flows. JFluidMech 1995; 286:229 255

[12] Issa RI, Solution of the implicitly discretised fluid flow equations by operator-splitting. JCompPhys 1986; 62:40 - 65

[13] Iwamoto K 2002; Database of fully developed channel flow. Tech. Rep. ILR-0201, Department of Mechanical Engineering, The University of Tokio

[14] Jasak H, Weller H, Gosman A, High resolution nvd differencing scheme for arbitrarily unstructured meshes. IntJNumerMethFluids 1999; 31:431 $-449$

[15] Keating A, Piomelli U, Balaras E, Kaltenbach HJ, A priori and and a posteriori tests of inflow conditions for large-eddy simulation. Physics of Fluids 2004; 16(12):4696 - 4712

[16] Klein M, Sadiki A, Janicka J, A digital filter based generation of inflow data for spatially developing direct numerical or large eddy simulations. JCompPhys 2003; 186:652 - 665

[17] Lee S, Lele S, Moin P, Simulation of spatially evolving turbulence and the applicability of Taylor's hypothesis in compressible flow. Phys Fluids 
A $1992 ; 4: 1521-1530$

[18] Lund TS, Wu X, Squires KD, Generation of turbulent inflow data for spatially-developing boundary layer simulations. J.Comp.Phys.1998; 140:233 - 258

[19] di Mare L, MKlein, Jones WP, Janicka J, Synthetic turbulence inflow conditions for large-eddy simulation. PhysFluids 2006; 18(2):025,107

[20] Pierce CD, Moin P, Method for generating equilibrium swirling inflow conditions. AIAA J 1998; 36:1325 - 1327

[21] Rhie C, Chow W, A numerical study of the turbulent flow past an isolated airfoil with trailing edge separation. AIAAJ 1983; 21(7):1225 - 1232

[22] Schlüter JU, Pitsch H, PMoin, Large eddy simulation inflow conditions for coupling with Reynolds-averaged flow solvers. AIAAJ 2004; 42(3):478 $-484$

[23] Schmidt S, McIver DM, Blackburn HM, Rudman M, Nathan GJ 2001; Spectral element based simulation of turbulent pipe flow. In: 14th Australasian Fluid Mechanics Conference, Adelaide University, Adelaide, Australia

[24] Smirnov A, Shi S, Celik I, Random flow generation technique for large eddy simulations and particle-dynamics modelling. JFluids Engineering 2001; $123: 359-371$

[25] Spalart PR, Numerical study of sink-flow boundary layers. J Fluid Mech 1986; 172:307

[26] Spalart PR, Direct simulation of a turbulent boundary layer up to $r_{\theta}=1410$. JFM 1988; 187:61 - 98

[27] Spalart PR, Watmuff JH, Experimental and numerical study of a turbulent boundary layer with pressure gradients. J Fluid Mech 1993; 249:337 $-371$

[28] Stefano GD, Vasilyev OV, Sharp cutoff versus smooth filtering in large eddy simulation. Phys of Fluids 2002; 14(1):364 - 369

[29] Tabor G, Weller HG, Large Eddy Simulation of premixed turbulent combustion using $\xi$ flame surface wrinkling model. Flow, Turbulence and Combustion 2004; 72:1 - 28

[30] Tabor G, Baba-Ahmadi MH, de Villiers E, Weller HG 2004; Construction of inlet conditions for LES of turbulent channel flow. In: Proceedings of the ECCOMAS Congress, Jyväskylä, Finland

[31] de Villiers E 2006; The potential for large eddy simulation for the modelling of wall bounded flows. $\mathrm{PhD}$ thesis, Imperial College

[32] Walpot R, van der Geld C, Kuerten J, Determination of the coefficients of langevin models for inhomogeneous turbulent flows by three-dimensional partical tracking velocimetry and direct numerical simulation. PhysFluids 2007; 19:045,102-1

[33] $\mathrm{Wu}$ X, Moin P, A direct numerical simulation study on the mean velocity characteristics in turbulent pipe flow. JFM 2008; 608:81 - 112 


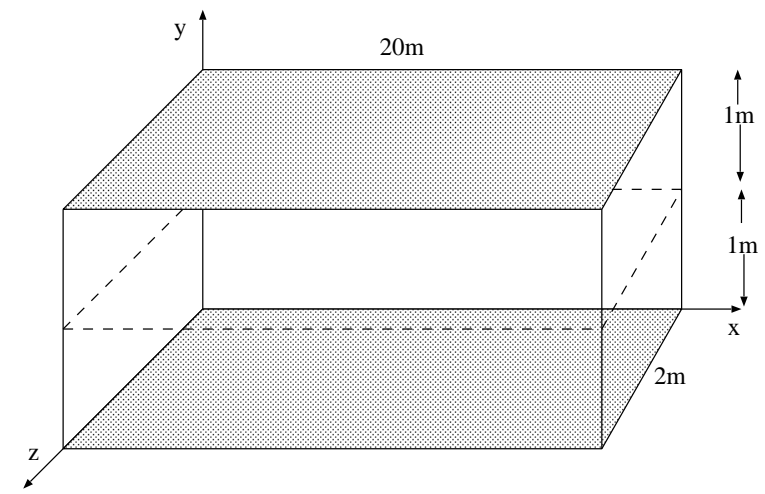

Fig. 1. Geometry for the channel; a $20 \mathrm{~m}$ long channel between two walls; flow is in the $x$-direction. 


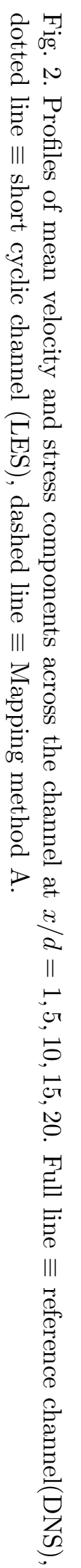
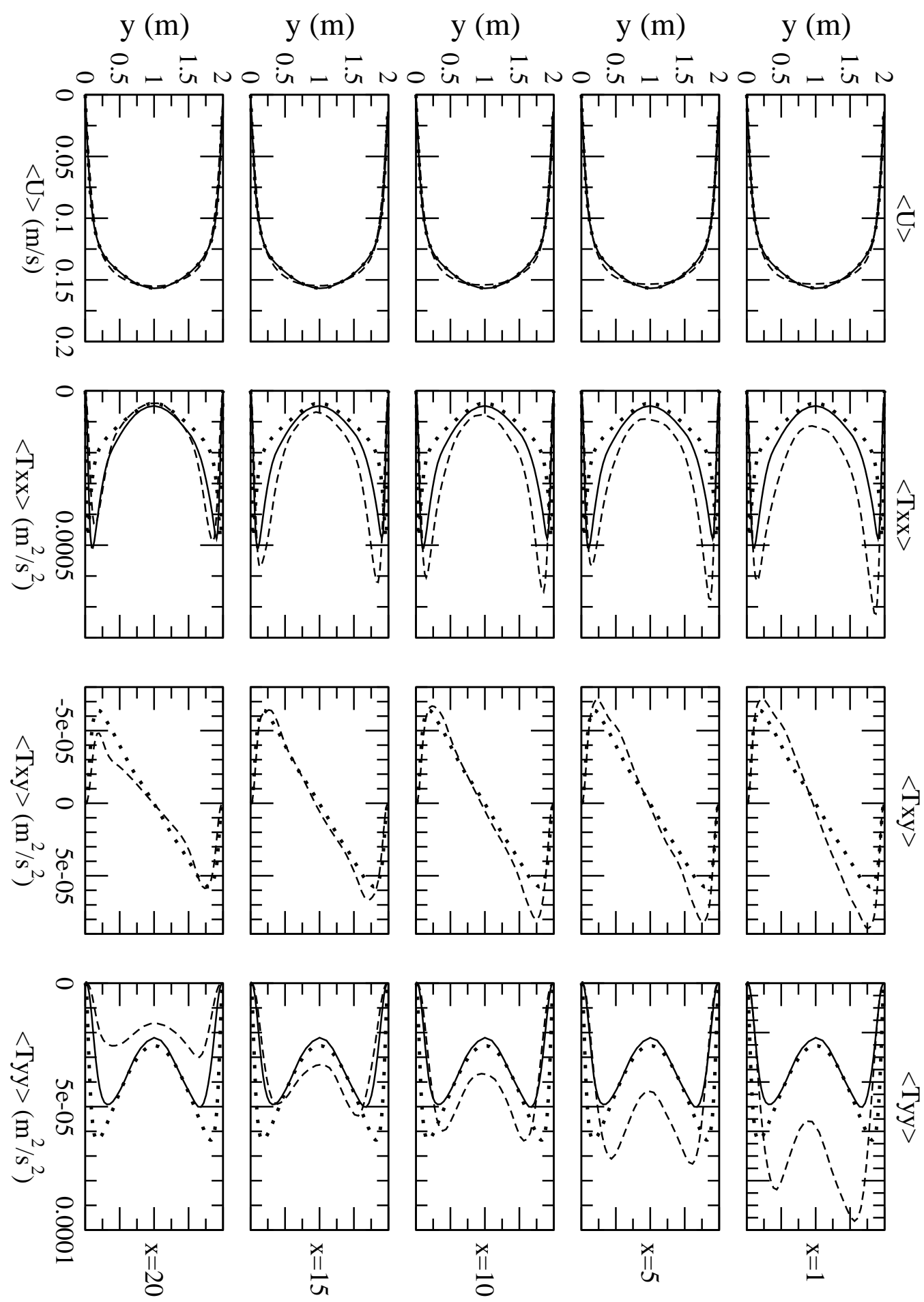


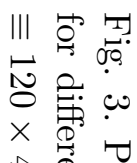

吕惫兽

N

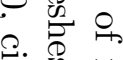

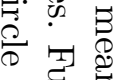

纪

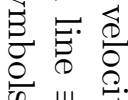

8

$\times$ 응

$\Delta$
$\times$
$x$

ㅇํㅇ

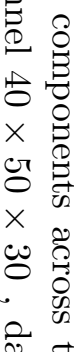

密客

है.

III

용

ç

$\times \leftarrow$

एن

०

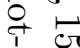

है

苛

III

g:

c

$\times$ 过
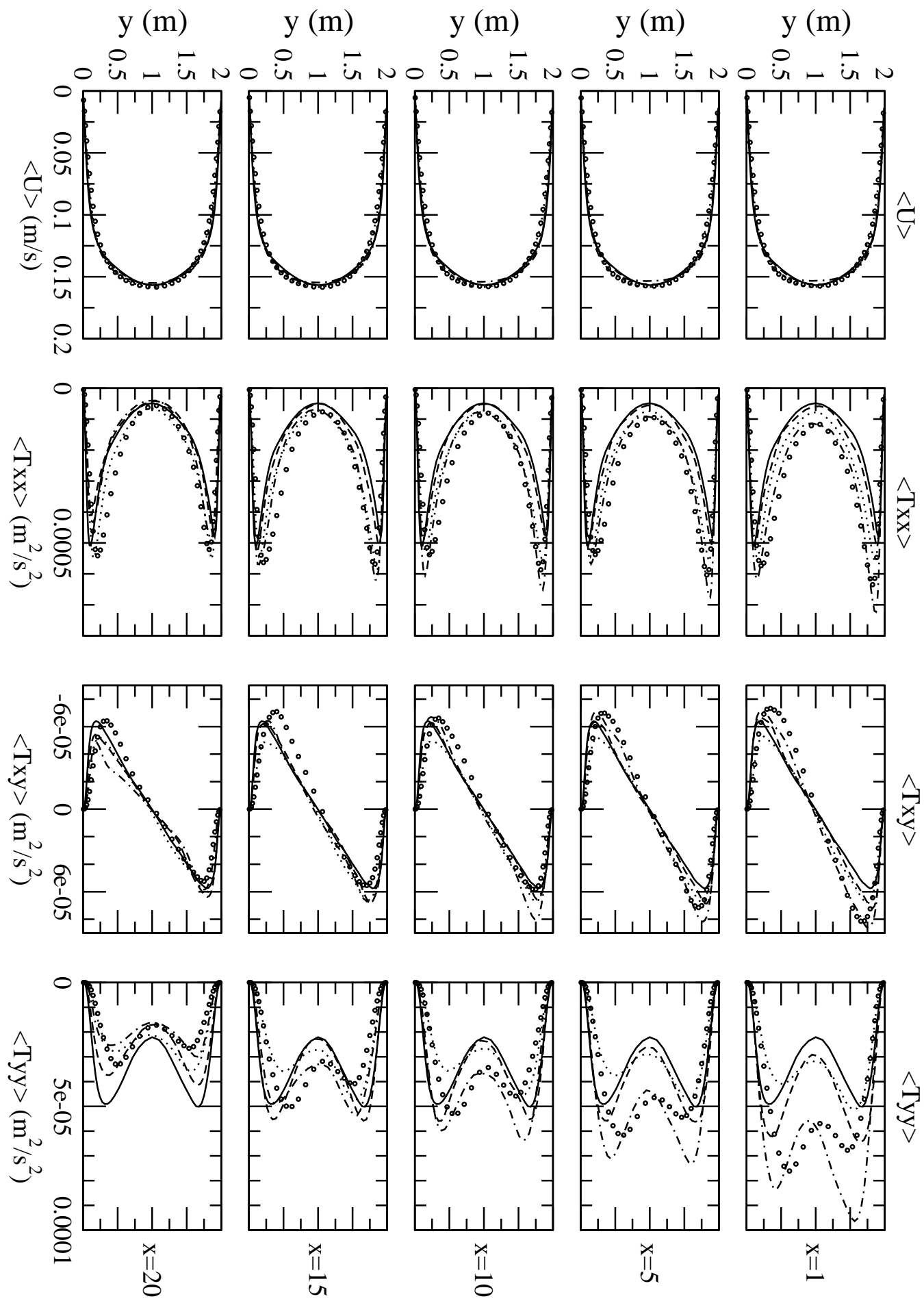

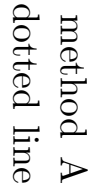


a.

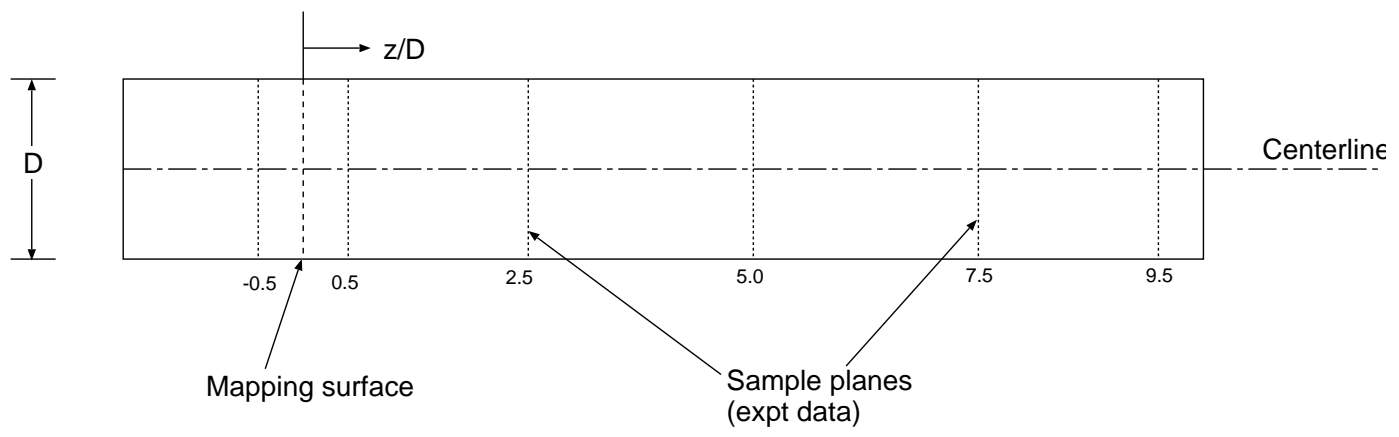

b.

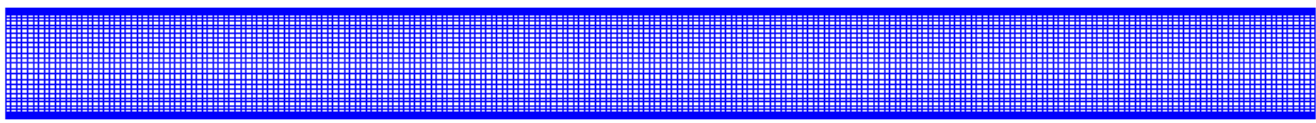

c.

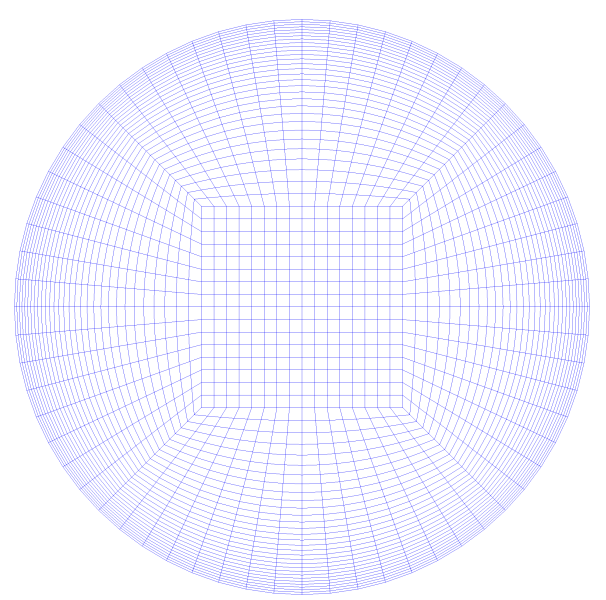

Fig. 4. Geometry for LES of the turbulent parallel pipe flow. Top figure (a). shows the geometry and block structure of the case, including the locations of the mapping surface and the sample locations. Centre figure (b). shows a cross-section of the mesh structure, and the bottom figure (c). an axial view of the mesh 


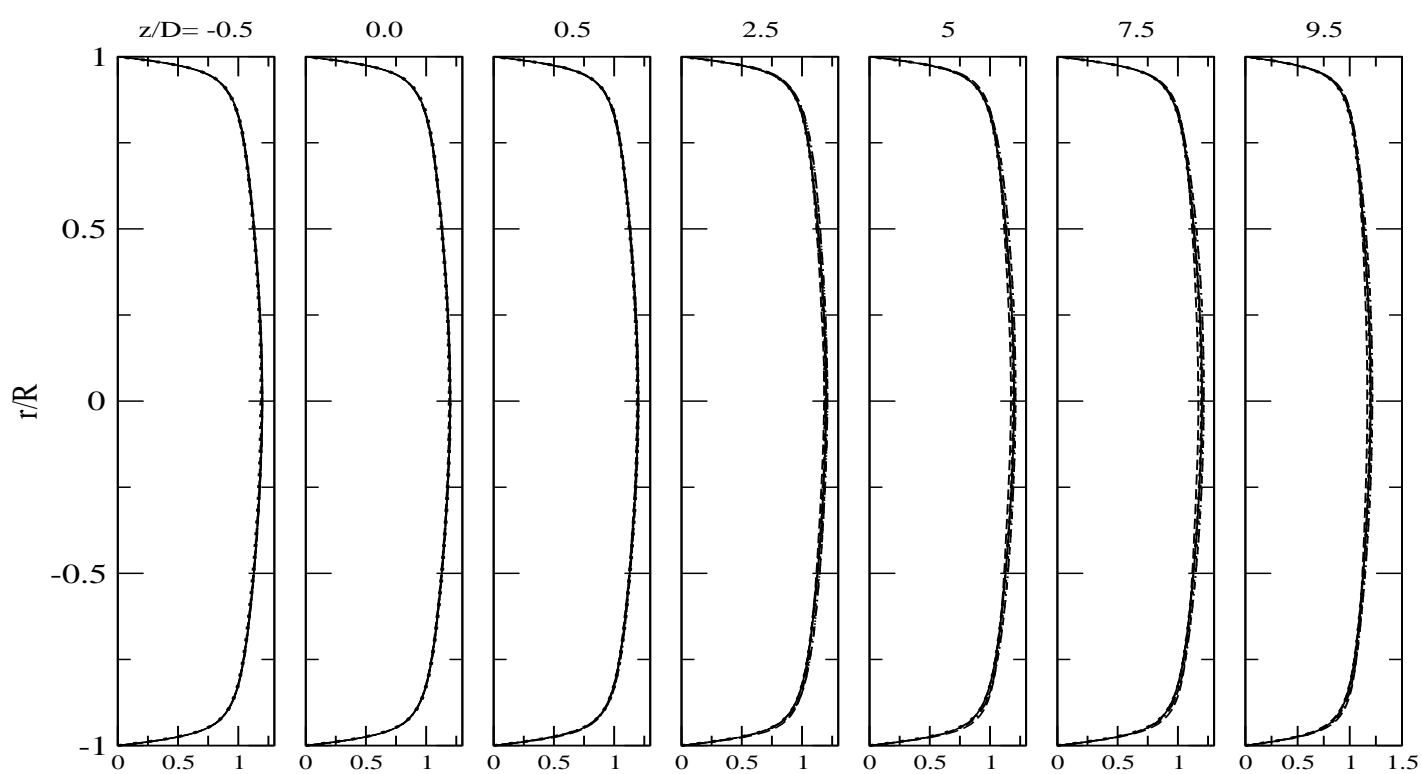

Fig. 5. Mean velocity profile $\widehat{u_{z}}$ at different distances from the inlet of the main domain. Full lines: Cyclic short Pipe, dotted lines: Method A, short dash lines: Method B, long dash lines: Method C, and double-dot-dash lines: Method D

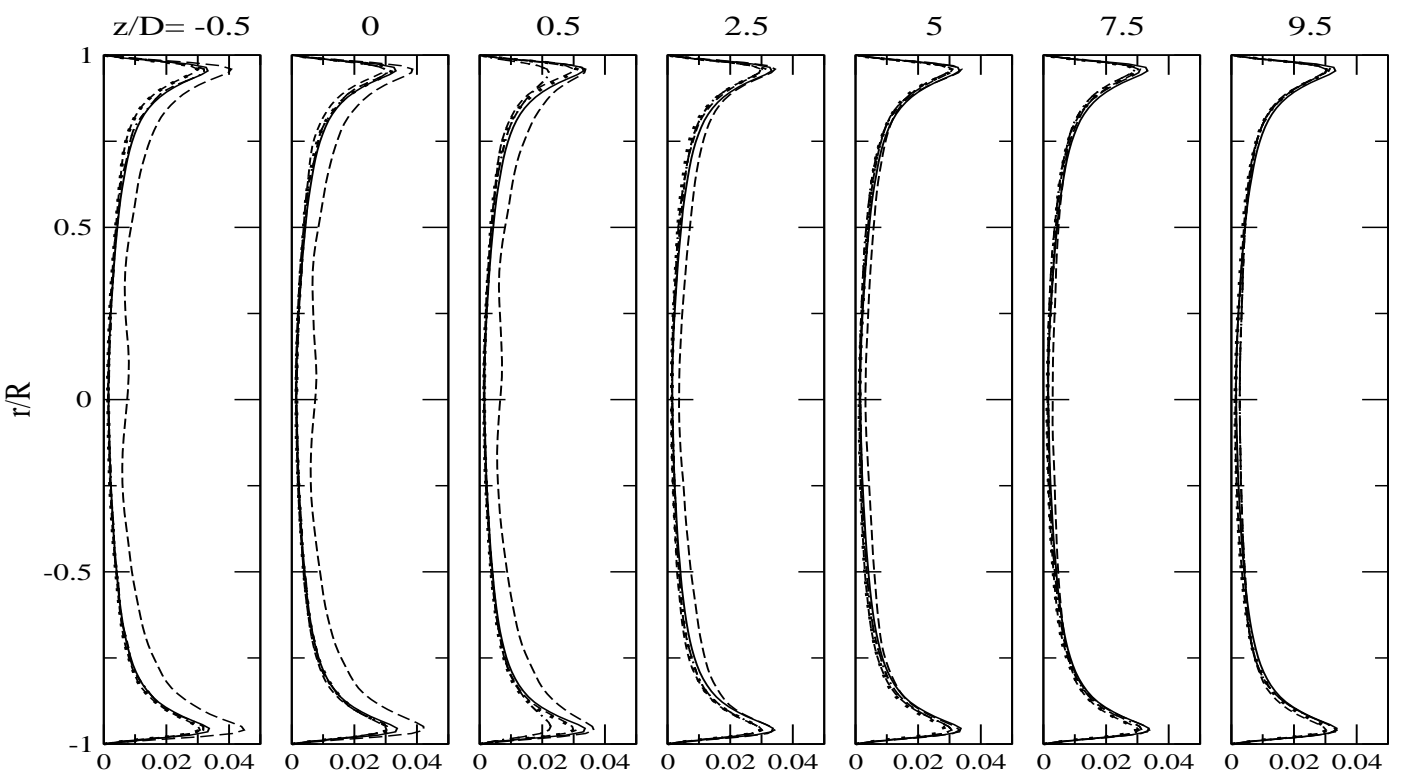

Fig. 6. Reynolds stress $\tau_{z z}$ at different distances from the inlet of the main domain. Lines are defined as in Fig. (5) 


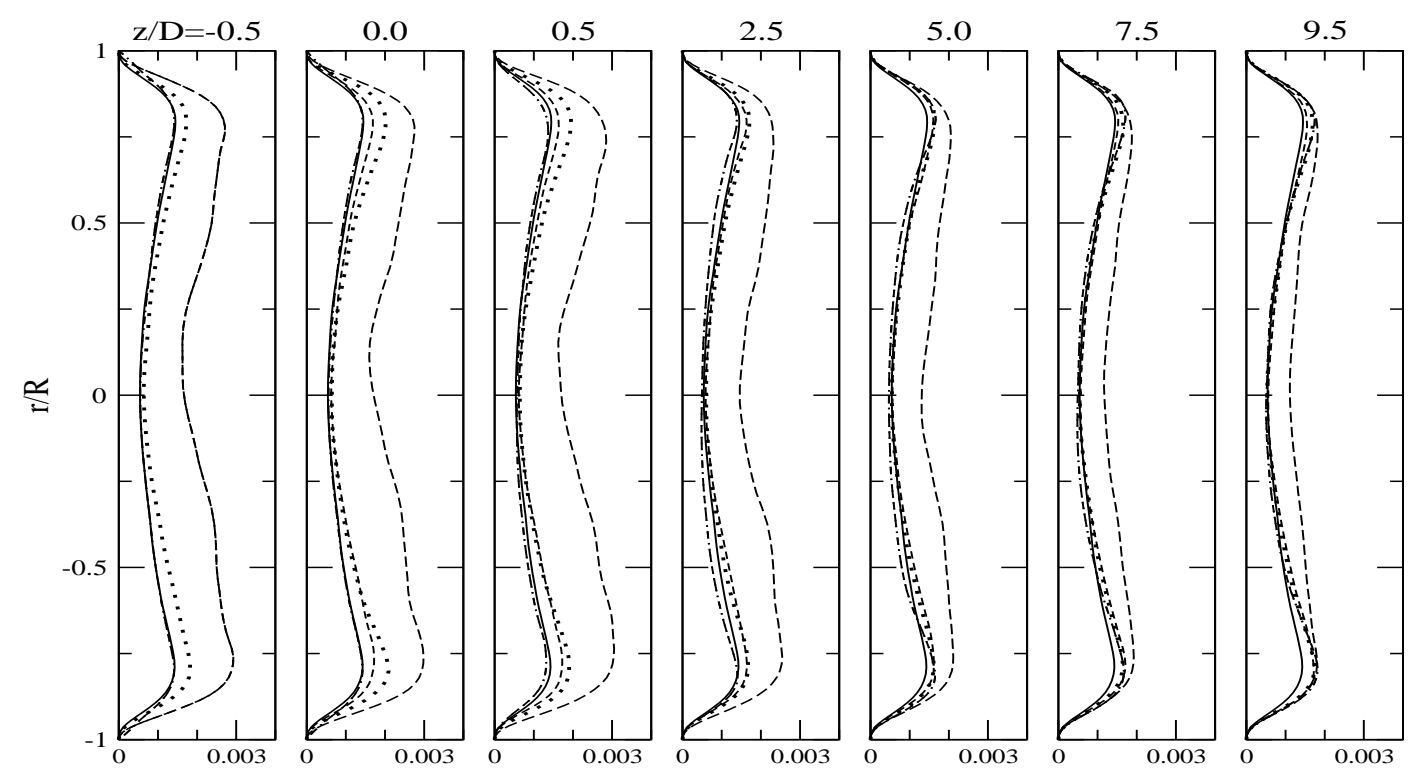

Fig. 7. Reynolds stress $\tau_{r r}$ at different distances from the inlet of the main domain. Lines are defined as in Fig. (5)

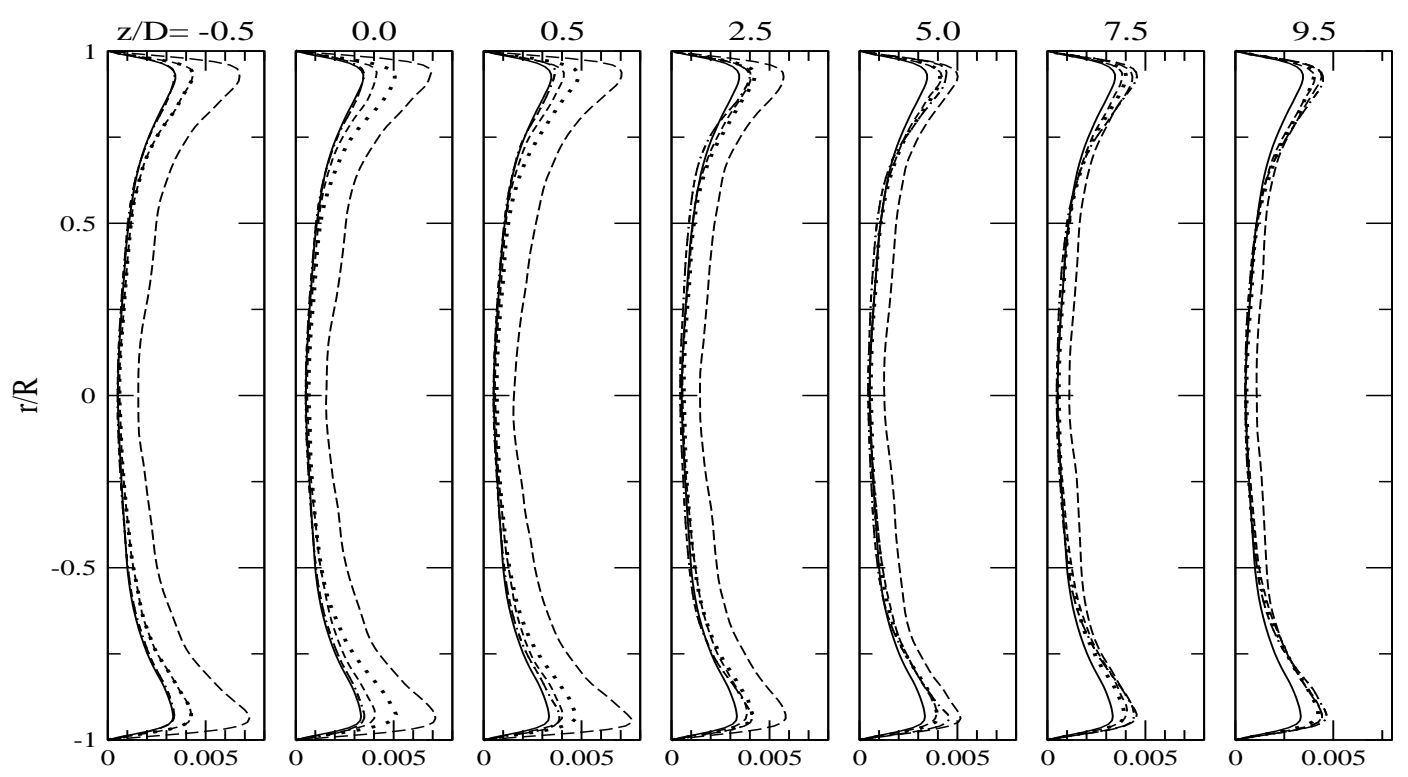

Fig. 8. Reynolds stress $\tau_{\theta \theta}$ at different distances from the inlet of the main domain. Lines are defined as in Fig. (5) 


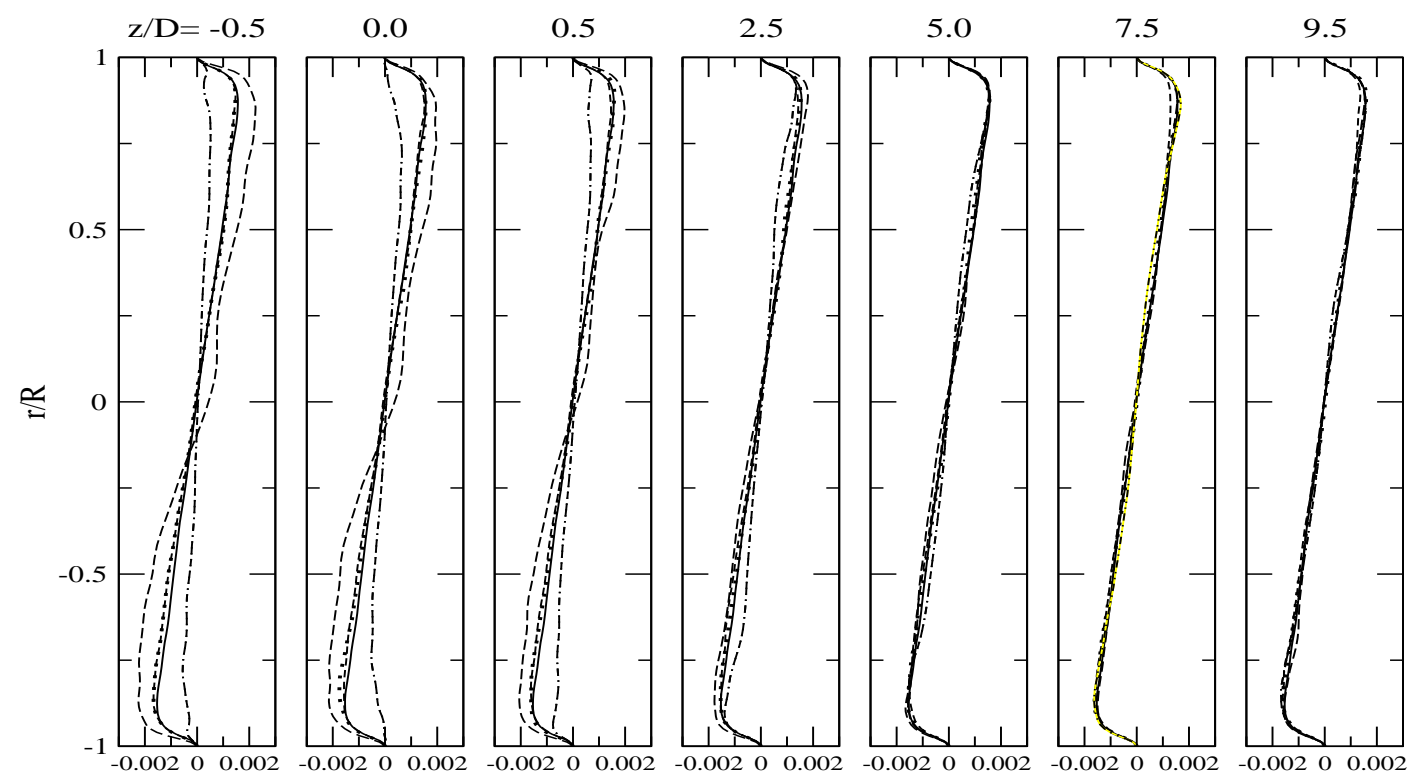

Fig. 9. Reynolds stress $\tau_{z r}$ at different distances from the inlet of the main domain. Lines are defined as in Fig. (5) 


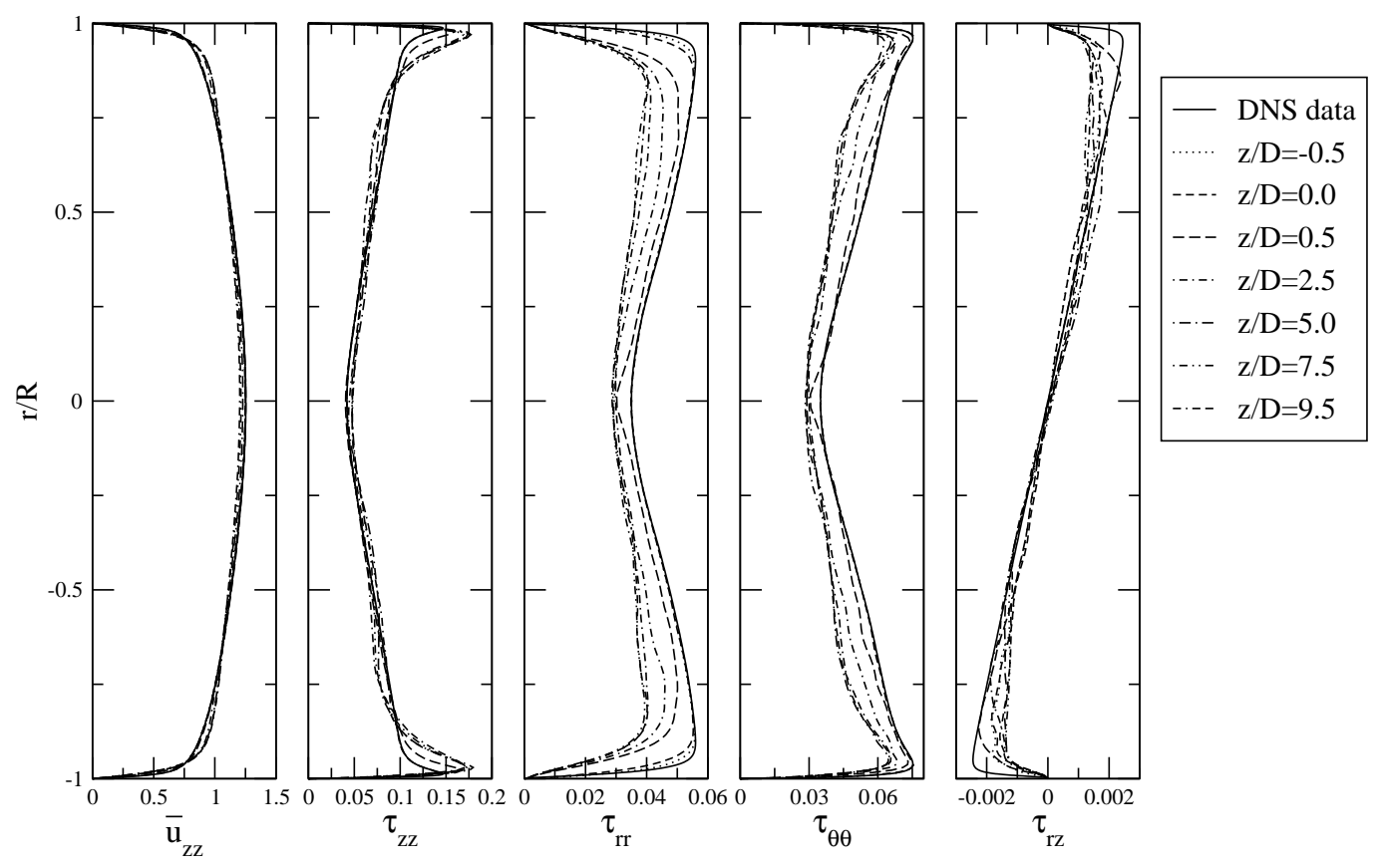

Fig. 10. Comparison of method D against DNS data [33] for $\mathcal{R} e=44,000$. Mean velocity and fluctuating components are shown at various points along the pipe $(z / D=0.0$ is the mapping plane). 


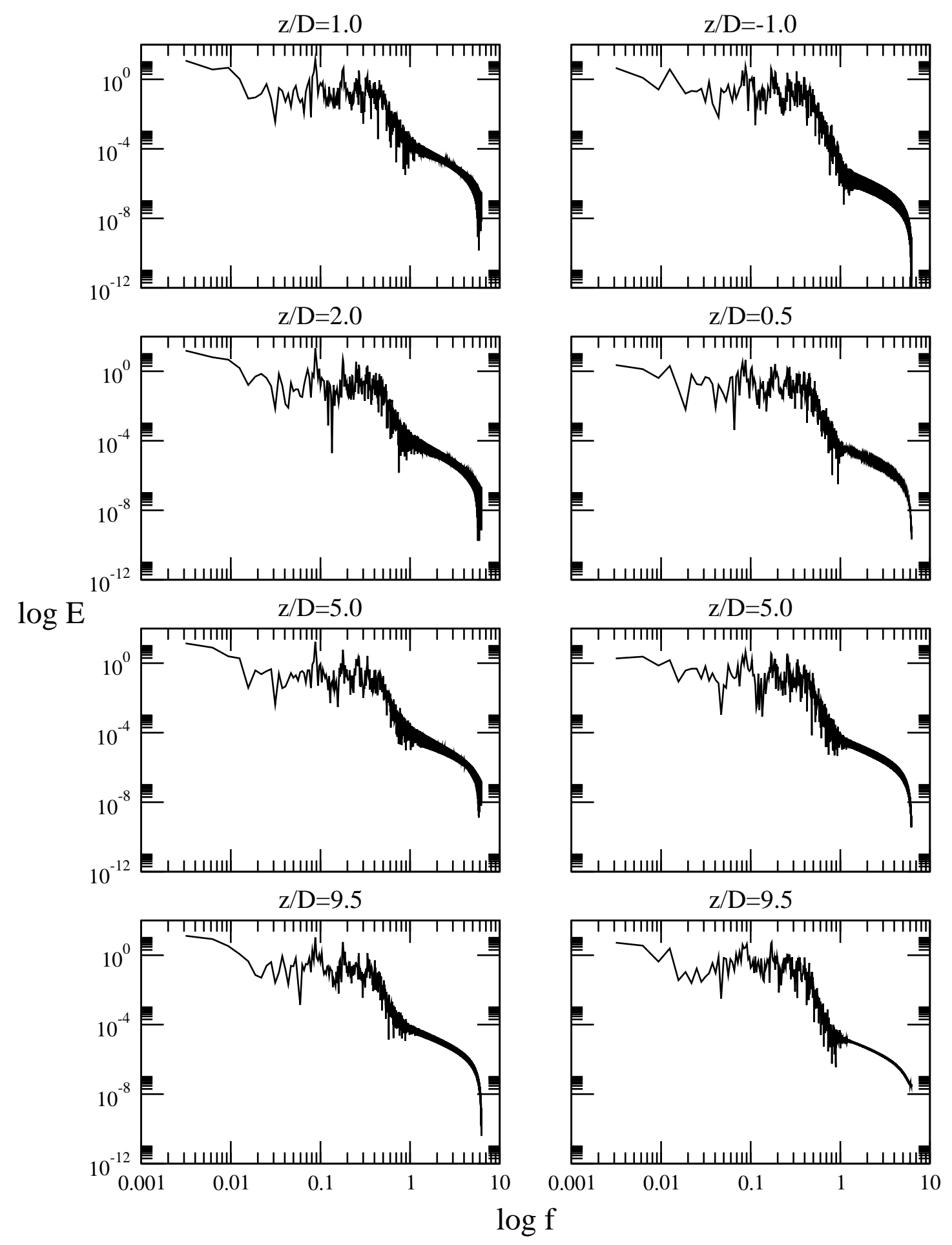

Fig. 11. Energy spectra generated from time series data at different distances from the inlet of the main domain. a) Left side figures: Method A, b) Right side figures: Method B. 


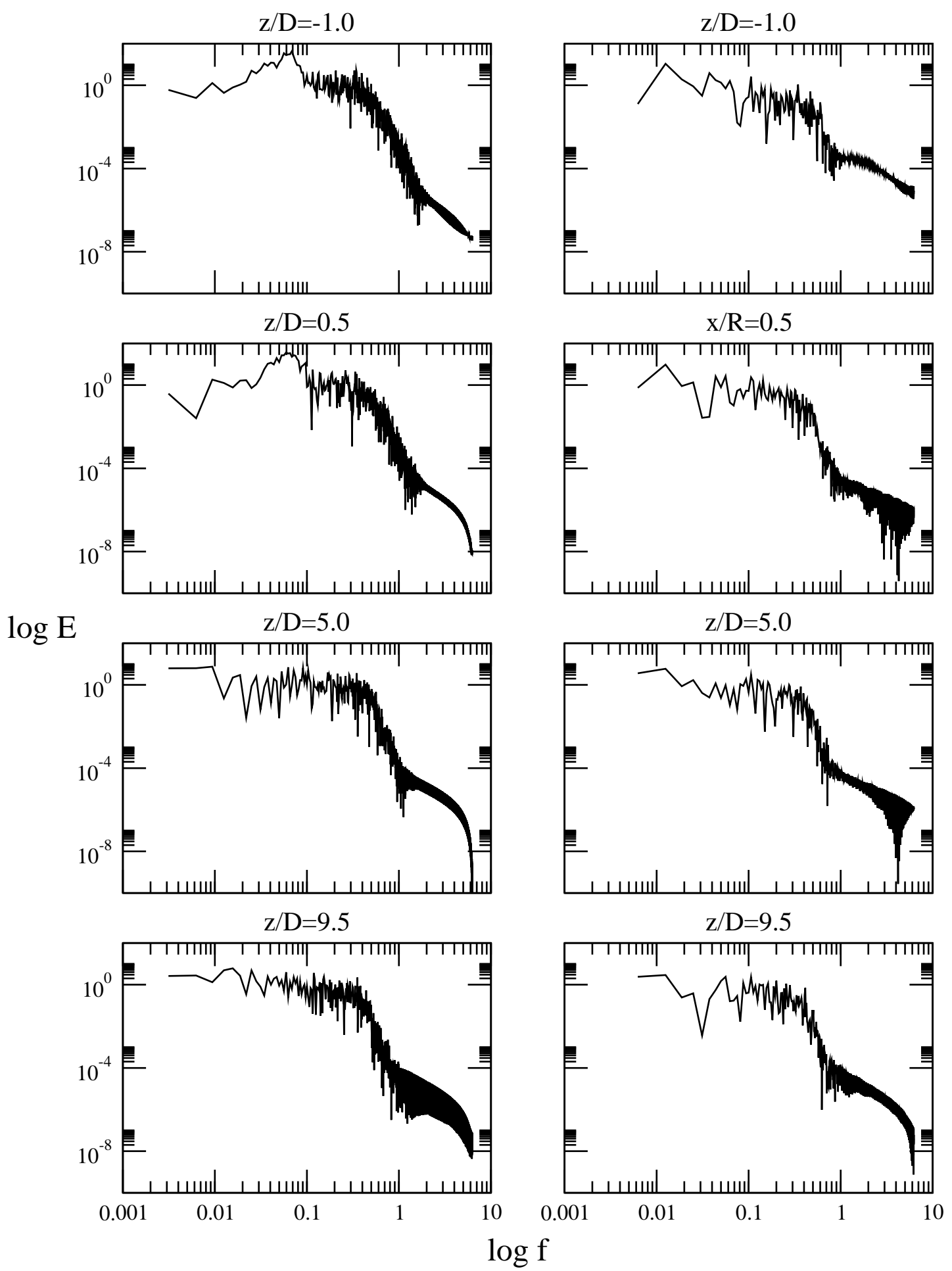

Fig. 12. Energy spectra generated from time series data at different distances from the inlet of the main domain. c) Left side figures: Method C, d) Right side figures: Method D. 
a.
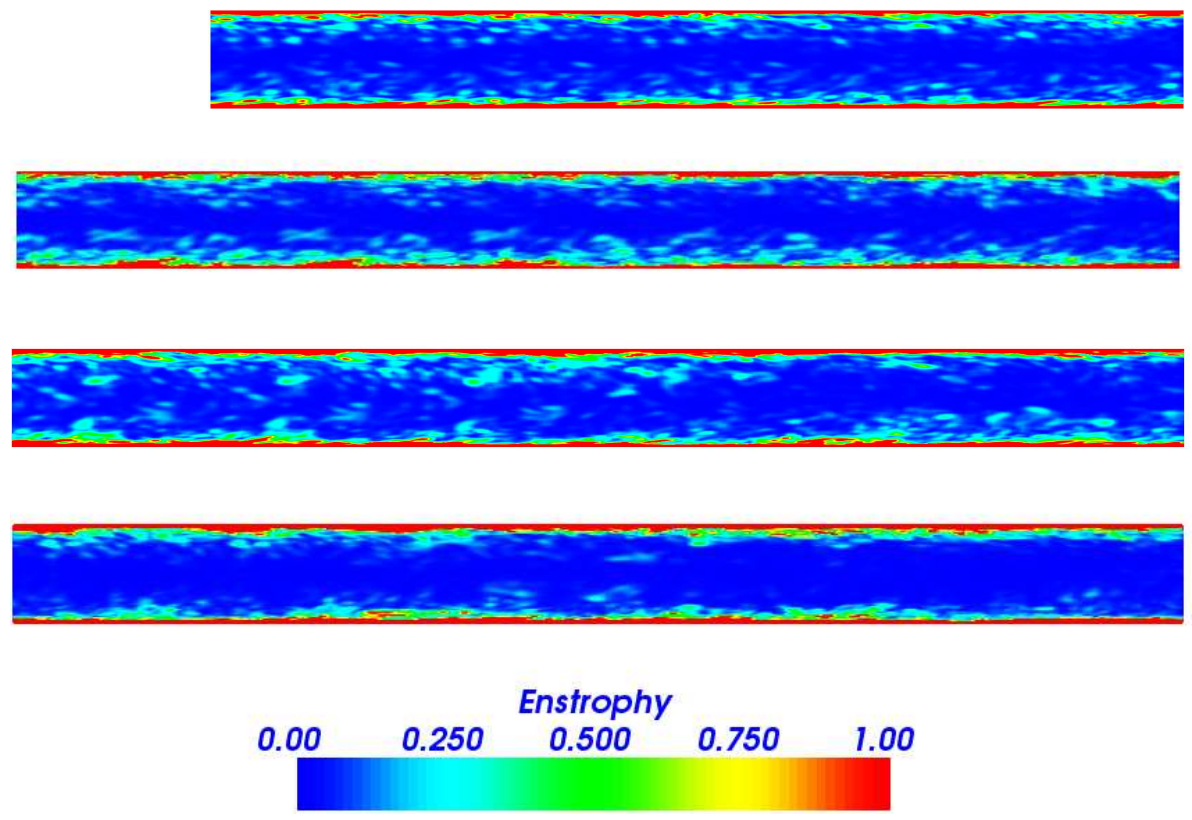

b.
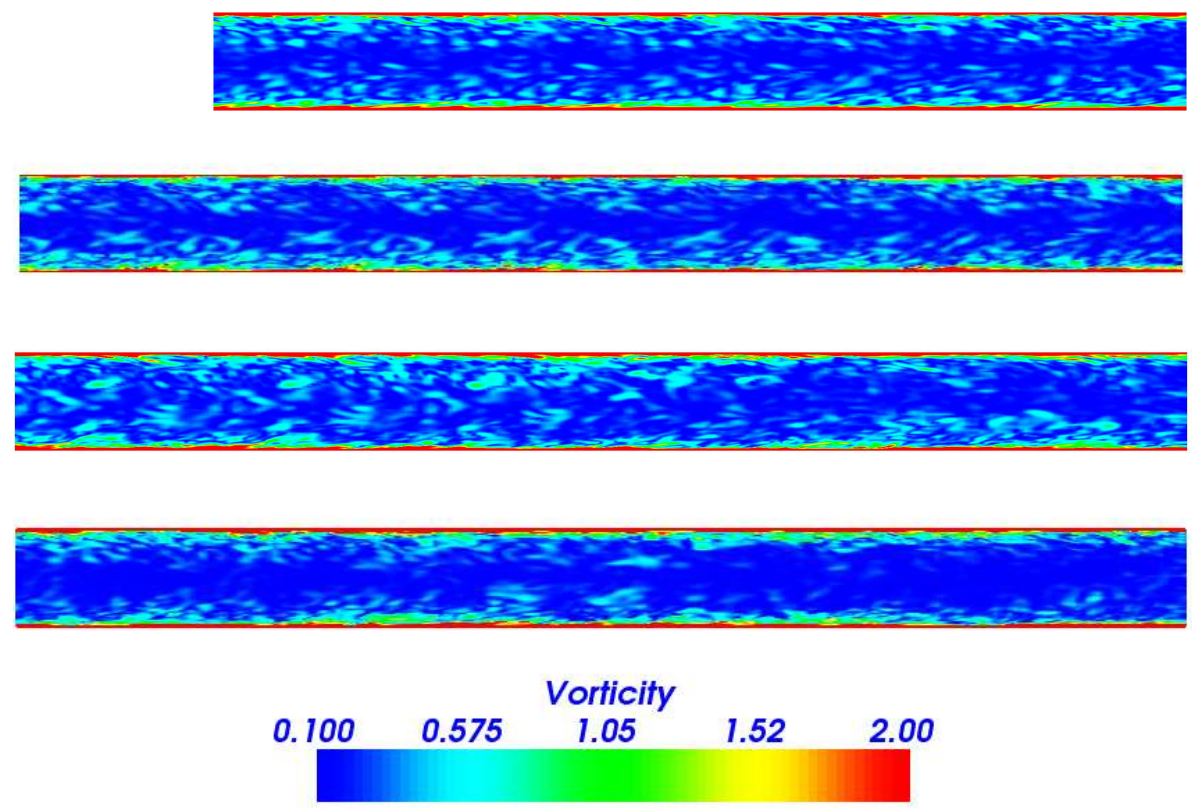

Fig. 13. a. Enstrophy plotted on the channel centre plane. b. Vorticity plotted on the channel centre plane. Downward from top: Methods A, B, C and D. 


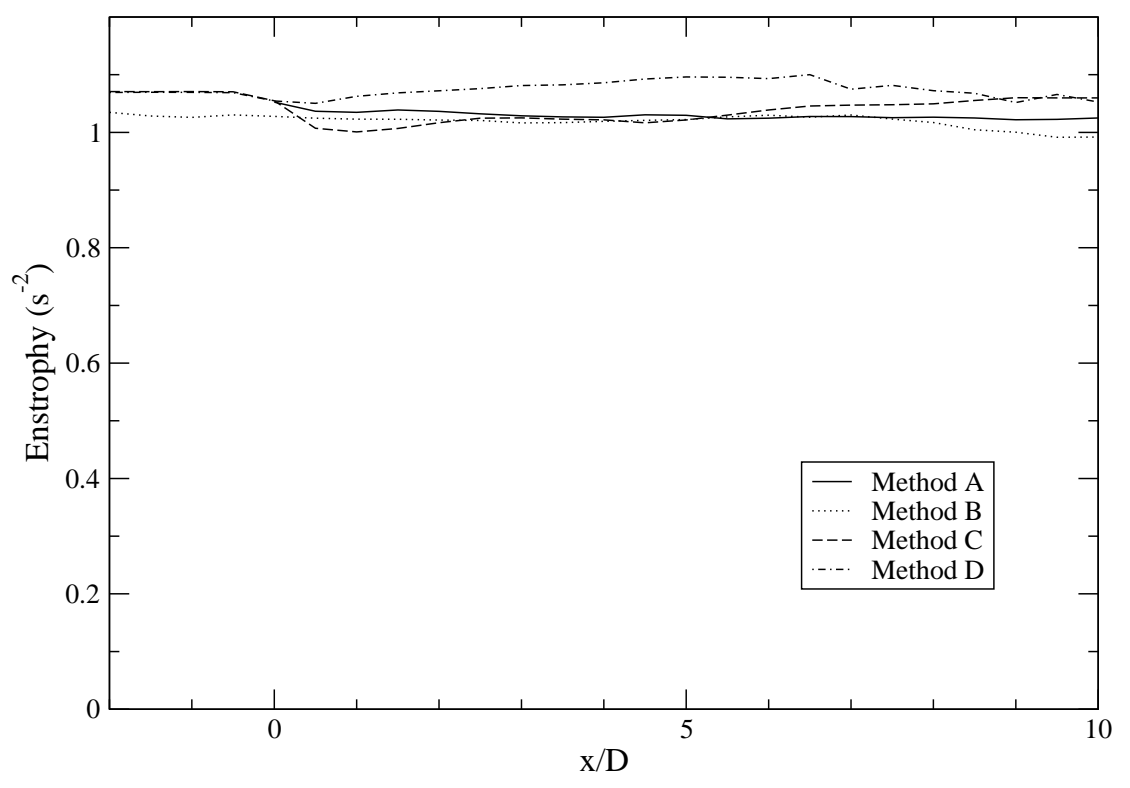

Fig. 14. Enstrophy changes along the channel length. Full line: Method A, Dotted line: Method B, Dashed line: Method C, and Dot/dash: Method D 